Cahiers de recherches médiévales

\title{
Jeanne aux panaches romantiques
}

\section{Françoise Michaud-Fréjaville}

\section{OpenEdition}

Journals

Édition électronique

URL : https://journals.openedition.org/crm/738

DOI : $10.4000 / \mathrm{crm} .738$

ISSN : 1955-2424

Éditeur

Honoré Champion

Édition imprimée

Date de publication : 1 juin 2005

Pagination : 259-272

ISSN : $1272-9752$

\section{Référence électronique}

Françoise Michaud-Fréjaville, "Jeanne aux panaches romantiques », Cahiers de recherches médiévales [En ligne], 12 spécial | 2005, mis en ligne le 28 juin 2008, consulté le 15 décembre 2022. URL : http:// journals.openedition.org/crm/738; DOI : https://doi.org/10.4000/crm.738 


\section{酷M}

\section{$-22-$ \\ Jeanne aux panaches romantiques}

On ne va presque jamais regarder la statue de Jeanne d'Arc dressée à Orléans sur la rive sud de la Loire. Qui peut aujourd'hui acheter au tabac du coin, sur la pittoresque place de la Bascule, une carte postale la représentant? Elle est introuvable, sinon en fouillant dans les boîtes des commerçants spécialisés en cartophilie, et là, même, qui se laisserait séduire par cette Jeanne au panache de bronze?

Pourtant, non seulement cette statue a une fort intéressante histoire propre qui n'est pas entièrement de mon propos direct aujourd'hui ${ }^{1}$, mais elle s'inscrit également dans le processus multiséculaire des représentations de l'héroïne dont nous avons fêté en 2001 , le $572^{\mathrm{e}}$ anniversaire des exploits orléanais.

À l'origine, Jeanne d'Arc aurait été portraiturée au naturel une fois, à Arras ou Reims, en attitude de messager, c'est ce qu'elle a dit elle-même ${ }^{2}$. Nous ignorons le destin de cette image et nous n'avons pas de trace d'une postérité iconographi$q^{3} e^{3}$. Ensuite, viennent les interprétations imaginaires des artistes, des commanditaires et, j'ose le dire, les fantasmes des amateurs qui voulurent voir Jeanne partout et suscitèrent beaucoup des fausses attributions et des faux qui encombrent encore la vue.

L'iconographie de Jeanne, jusqu'à la révolution de 1837, c'est-à-dire jusqu'à la statue de Marie d'Orléans, découle de deux «modèles». Le premier fut le monument en bronze élevé au XVI ${ }^{\mathrm{e}}$ siècle sur le pont des Tourelles, avec Jeanne de profil, à genoux, tête nue et en armure ${ }^{4}$. Le second, à la postérité abondante, a été la «Jeanne des échevins» du Musée Historique ${ }^{5}$, une Judith germanique retouchée ${ }^{6}$, en

${ }^{1} \mathrm{~F}$. Michaud-Fréjaville «Métamorphoses et permanences de la statuaire publique française célébrant Jeanne d'Arc ", Mémoire sculptée de l'Europe, Colloque international, Strasbourg, décembre 2001, sous presse.

${ }^{2}$ Procès de condamnation, éd. P. Champion, Paris, 1920, t. I, p. 75 : «Interrogée s'elle avait point veu ou fait faire aucuns ymaige ou paincture d'elle et a sa semblance : respond qu'elle vit a Arras une paincture d'elle en la main d'un Escot, et y avoit la semblance d'elle toute armee, et presentoit une lectre a son roy, et estoit agenoullee d'un genoul. Et dit que oncques ne vit ou fist faire autre ymaige ou painture a la semblance d'elle» (version du manuscrit d'Urfé). Le père Doncœur a décelé une erreur de graphie sur le nom de la ville: ce serait Reims ; mais cela ne change rien à notre propos.

${ }^{3}$ On peut cependant noter que le «portrait» cinématographique de Besson (1999) s'intitule The messenger.

${ }^{4}$ Mgr. P. Brun, «Le premier monument à Jeanne d'Arc sur l'ancien pont d'Orléans », Dossiers de l'Archéologie, $\mathrm{n}^{\circ}$ 34, mai 1979, p. 71-76.

${ }^{5}$ Musée Historique et Archéologique de l'Orléanais, inv. A 6922. D. Ojalvo, «Les deux portraits de Jeanne d'Arc du Musée historique de l'Orléanais », Bulletin de la Société Archéologique et Historique de l'Orléanais (B.S.A.H.O.), $\mathrm{n}^{\circ} 49,1979, \mathrm{p} .142$, avec une inversion des titres des reproductions.

Cahiers de Recherches Médiévales (XII -XV S.), 12spé, 2005 
robe décolletée à taille haute, un béret à cinq plumes sur la tête, aux rubans noués sous le menton, l'épée pointe en l'air (geste de la Justice rendue) à la main droite et un «mouchoir» pendant pesamment de la main gauche (il s'agit sans doute dans un prototype non-johannique du poids de la tête d'Holopherne). Le portrait des échevins éludait largement l'aspect guerrier, en dépit de l'arme dont les épigones d'un peintre inconnu chargèrent d'ailleurs parfois la main gauche ${ }^{7}$ ! C'est de la postérité de cette étrange image de guerrière en costume de cour dont il sera désormais question ici, tant elle fut popularisée par des séries de gravures ${ }^{8}$.

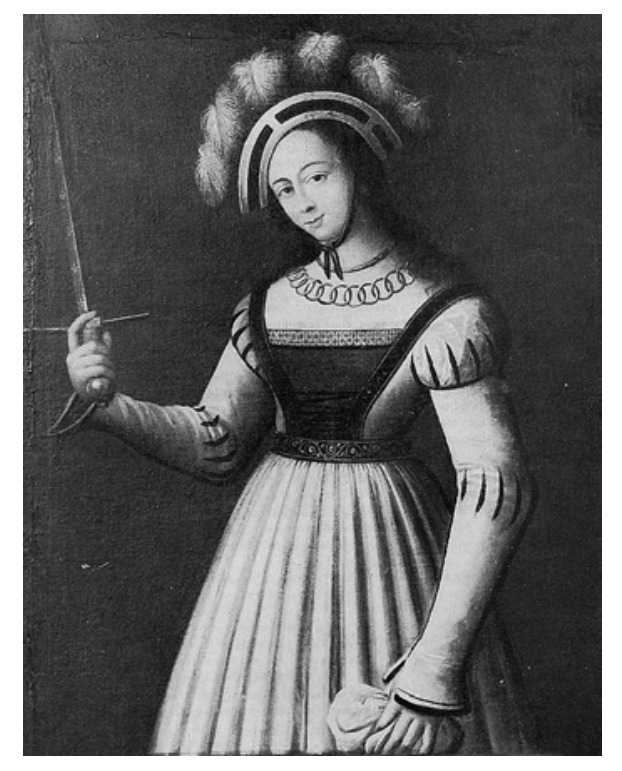

La Jeanne du «tableau des échevins » (1581)

${ }^{6} \mathrm{O}$. Bouzy, «Images bibliques à l'origine de l'image de Jeanne d'Arc», Images de Jeanne d'Arc, Actes du colloque de Rouen, Jean Maurice et Daniel Couty dir., P. U. F., 2000, p. $237-$ 242.

${ }^{7}$ C'est le cas du Portrait des échevins de la version conservée dans l'Hôtel Groslot d'Orléans : le peintre a gardé à la main droite son geste primitif qui semble maintenant désigner la lame de l'épée, tenue désormais dans la main gauche.

${ }^{8}$ En particulier celle de Lemire, dédicacée à madame de Cypierre en 1774 (.Images de Jeanne d'Arc, Catalogue de l'Exposition de l'Hôtel de la Monnaie, Paris, 1979, p. 65, n 72. 


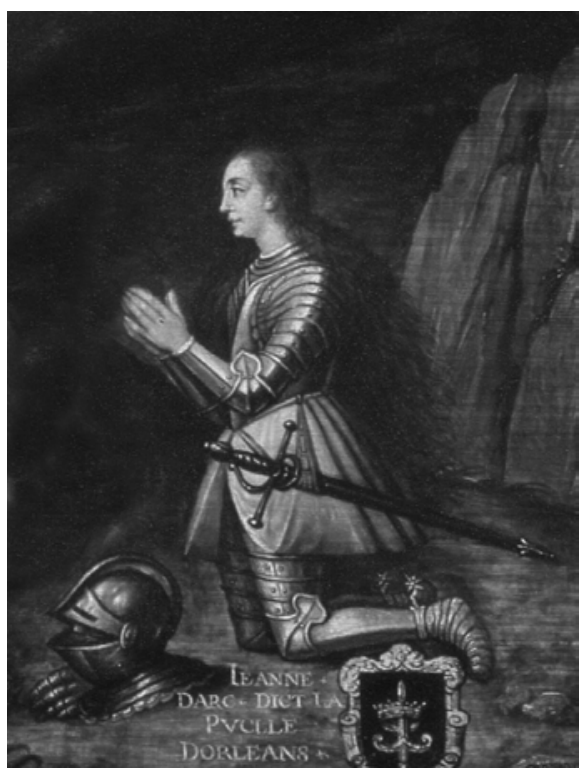

La Jeanne du «monument du pont ». Détail du tableau de Quesnel (1723)

La féminité hardie qui se dégageait de cette tête prise isolément a tenté les imitateurs. Une des premières représentations modernes est la mutation révolutionnaire gravée par J. Voyer, en $1795^{\circ}$ : les cheveux se frisent sur le front, le béret devient un bonnet sur lequel court une couronne de lauriers, les plumes se recourbent telle la pointe de la coiffure phrygienne, la robe aux manches à crevés fait place aux plis de la tunique antique, découvrant des bras nus. L'épée est remise à un fourreau invisible accroché à un baudrier qui fait valoir une fière poitrine. De circonstance, cette Jeanne de Thermidor n'aura pas de postérité. En revanche, le détail de la tête des «échevins", inversé ou dans le bon sens, a été reproduit dès le début du $\mathrm{XIX}^{\mathrm{e}}$ siècle à des centaines d'exemplaires. Selon le talent des dessinateurs et des graveurs, les résultats sont plus ou moins convaincants. Après un dessin d'Isabey et son interprétation par Boilly, gravée par Motte pour le Nouveau journal des Dames et des Demoiselles de $1821^{10}$, on peut suivre jusque vers 1835 les mutations d'une image rapetissée ou agrandie ${ }^{11}$, affinée, christianisée, ${ }^{12}$ remarquable surtout par un regard indifférent, mutin ou intérieur.

${ }^{9}$ Images de Jeanne d'Arc, Catalogue..., op. cit., p. 68, n ${ }^{\circ} 78$, .

${ }^{10}$ C. J. A., inv. 00.173. 136

${ }^{11}$ C. J. A., inv. 00.173.078, 3 x 5 cm. ; inv. 00.173.077, 6 x $6.8 \mathrm{~cm}$ (Delpech et Bayard) ; inv. 00.173. 809 X $10.5 \mathrm{~cm}$ (par Audibran).

${ }^{12}$ C.J.A., inv. 00.173.137, 6 × $7.5 \mathrm{~cm}$, version non signée, au fin visage, Jeanne porte au cou une croix de Jeannette et lève les yeux au ciel. 


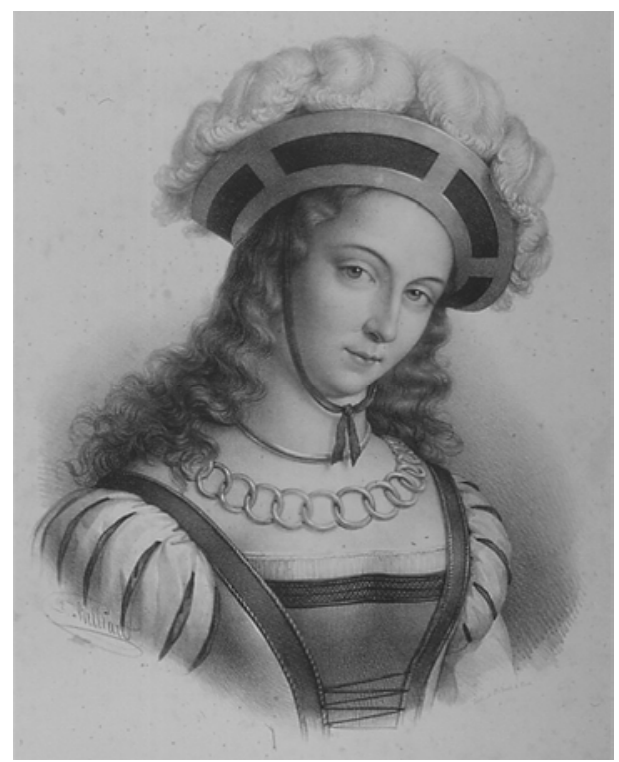

Un portrait copié (inversé) sur le « tableau des échevins » d'Orléans Dessin de Baillard, lithographie de Delpech à Paris (C.J.A. 00.173.007)

La première interprétation fut sans doute sa traduction en trois dimensions grâce au buste de la fontaine de Domrémy. La représentation la plus intéressante combine l'œuvre sculptée de Legendre-Héral (1820) avec une allégorie de la Restauration, clairement royaliste, où l'on voit la France couronnant ce buste curieusement juché sur le linteau de la maison de Domrémy, au-dessus des tisons du bûcher ${ }^{13}$.

Les frontispices et culs-de-lampe de divers opuscules, dont seuls sont conservées le portrait au béret devenu une icône, permettent de mesurer l'usure des planches et le goût du public de la Restauration et de la Monarchie de juillet qui appréciaient sans doute l'immuabilité d'une image restée telle qu'au temps des Valois...

${ }^{13}$ L. Jollois, Histoire abrégée de la vie et des exploits de Jeanne d'Arc [...], Paris Kilian, 1821, frontispice, dessin de Lafitte, gravé par Schroeder (C. J. A., inv. 74.12.2649). La symbolique se complique de deux angelots tenant l'un l'étendard aux fleurs de lys et l'autre l'épée et l'écu de Jeanne; une colonne brisée porte les noms des villes d'Orléans, Gergeau, Beaugency, Patay, Troyes, Rheims, le sacre étant la justification de l'épopée johannique. Rappelons néanmoins que Louis XVIII $(† 1824)$ ne se fit pas sacrer. 


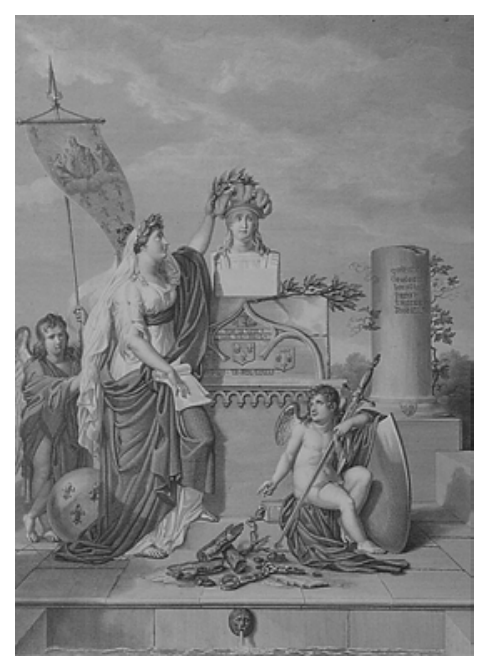

La France couronnant le buste de Jeanne d'Arc

Dessin de Lafitte, gravé par Schroeder et Gellée (C.J.A., inv. 74.12.2649)

Après la tête, le corps. Le «portrait des échevins » était hiératique, coupé aux genoux, masqué par un cartel suffisamment énigmatique pour qu'on ne sache s'il était contemporain de la peinture ou plaqué habilement sur une Judith de réemploi. Il fallait cependant que Jeanne eût des jambes. Elle en avait acquis dans la "Galerie des hommes illustres », peinte pour Richelieu (par Philippe de Champaigne, croit-on, et aujourd'hui disparue) et vulgarisée par la gravure, la tapisserie, Vouet et Vignon. Un exemple en est donné dans les Portraits des illustres françois de Vulson de la Colombière $(1668)^{14}$. Outre le mouvement de la jupe qui trahit une marche décidée, la nouveauté est ici l'introduction d'une cuirasse visible qui, sans enlever les crevés des manches ni les mailles du collier d'or, militarise vraiment la douce pucelle et permet de faire la transition non vers Boilly mais vers Gois et d'autres panaches romantiques.

${ }^{14}$ C.J.A., inv. 00.173.147, 7.5 x $13.5 \mathrm{~cm}$. Extrait de Vulson de la Colombière, Les portraits des hommes illustres françois/qui sont peints dans la gallerie du Palais/ Cardinal de Richelieu [...], Paris, François Mauger, 1668. Cf. Images de Jeanne d'Arc, Catalogue..., op. cit., p. 46, $\mathrm{n}^{\circ} 31$. 


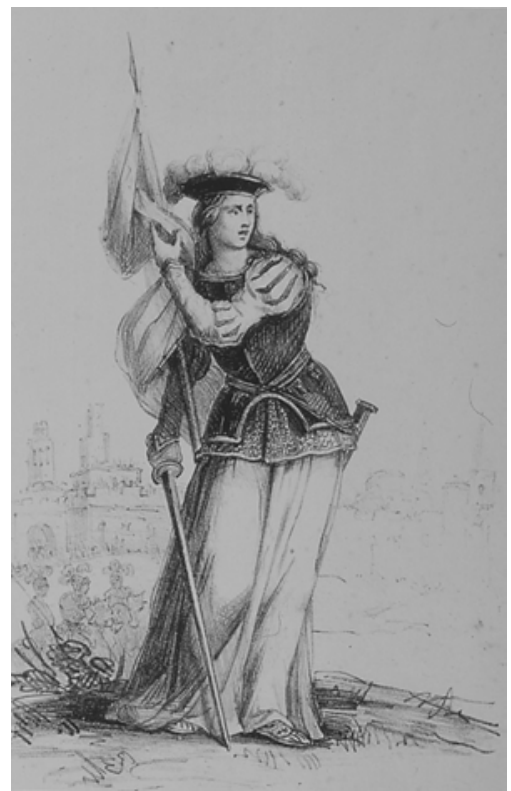

La Pucelle militarisée

Dessin de Chabriac lithographié par Bernard (C.J.A., 00.173.146)

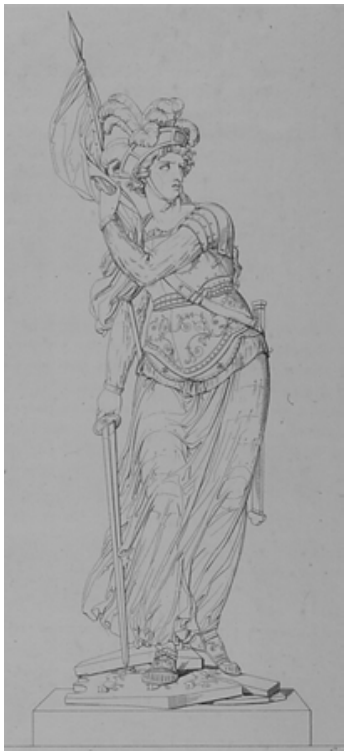

Projet pour une statue de Jeanne d'Arc

Dessin de Gois fils, gravé par C. Normand (C.J.A., 01.027.007) 
En 1803, au salon, on remarqua en haut lieu, c'est-à-dire le premier consul lui-même, la maquette d'Evariste Gois, fils d'un sculpteur terminant alors une carrière honorable, et qui lui-même avait séjourné à Rome. À la suite de tractations entre les édiles, le préfet Maret et l'artiste, une maquette fut présentée aux fêtes de la délivrance de 1803 et l'érection, après souscription, eut lieu en $1804^{15}$. La meilleure vue est celle du prospectus dans sa clarté linéaire ${ }^{16}$. Elle nous montre les détails de ce que nous ne regardons plus guère aujourd'hui sur l'original. Le costume est des plus composites, on reconnaît le béret et le décolleté carré du portrait des échevins, mais aussi les cheveux très agités d'Isabey et Boilly ; la cuirasse à la Romaine et la longue jupe viennent de Philippe de Champaigne; les plates des jambières et leurs rivets se devinent sous une robe du type des linges mouillés de la sculpture néo-classique, les écrevisses des épaulières continuées par la cotte de maille ont remplacé les crevés des manches civiles. La pose est néanmoins plus originale que le modèle du XVII siècle: l'étendard, oublié depuis le dessin de Vignon pour la Galerie des femmes fortes $(1647)^{17}$, fait sa réapparition. Le drapeau depuis la Révolution était devenu un élément fondamental de la rhétorique politique et militaire : le tricolore avait remplacé le blanc, et les faisceaux des prises sur les régiments commençaient à peupler les murs des Invalides. Jeanne foulait ici les léopards de l'écu anglais en serrant contre elle un drapeau pris à l'ennemi ${ }^{18}$ et non la bannière avec le Dieu de l'Apocalypse et l'inscription Jhesus Maria, marques chrétiennes encore mal venues en 1803, malgré le concordat de 1801. La statue prit place au Martroi, mais elle ne faisait guère de volume ( $2 \mathrm{~m} 60$ de haut sur un piédestal de $2 \mathrm{~m} \mathrm{90)}$ bien qu'on ait pris soin de la placer non pas au centre mais dans le resserrement oriental de la place (ou «petit Martroi ») et fut rapidement dépréciée : en 1829, un feuilletoniste pouvait la dauber sans crainte d'émouvoir les lecteurs orléanais : "une petite statue de chambre, montée sur un petit piédestal, entourée d'une petite grille ; tout cela mal tenu; les dalles du pavé cassées et disjointes par l'herbe qui les encadre de tous côtés : voilà le monument. Cette statue dans un musée pourrait être d'un joli effet [...] Cette image est celle d'une guerrière quelconque, ce pourrait être Clorinde ou Bradamante $^{19}$ tout aussi bien que Jeanne d'Arc. En vain l'épée abaissée semble annoncer que jamais elle ne répandit le sang; rien ne révèle le caractère religieux, enthousiaste, inspiré de cette femme extraordinaire. Je voudrais qu'on l'eût repré-

\footnotetext{
${ }^{15}$ Sur le sens politique de la statue de Gois, on peut consulter l'article intéressant, mais dont je ne partage pas certaines affirmations, de N. M. Heiman, "The art of politics in early nineteenth century France : E.-É. Gois's Jeanne d'Arc pendant le combat as metaphor ", Gazette des Beaux-Arts, Juillet-août 1998, p. 29-46.

${ }^{16}$ Gravée par C. Normand pour le salon de 1803 . Arch. mun. Orléans, 8 M 18. Voir aussi une version plus simple encore dans Images de Jeanne d'Arc, catalogue... op. cit., p. 71, $\mathrm{n}^{\circ} 80$.

${ }^{17}$ Images de Jeanne d'Arc, Catalogue... op. cit., p. 47, $\mathrm{n}^{\circ} 33$ : dessin original de Vignon pour la Galerie des femmes fortes de Lemoyne.

${ }^{18}$ « J'ai représenté notre héroïne dans le moment ou elle délivre la ville d'Orléans et arrache un drapeau aux ennemis », explique Gois lui-même dans son projet manuscrit de prospectus pour la souscription du monument, projet fortement remanié par la commission des édiles orléanais. Arch. mun. Orléans, 8 M 18.

${ }^{19}$ Respectivement héroïnes guerrières de la Jérusalem délivrée du Tasse et du Roland furieux de l'Arioste.
} 
sentée après la victoire, en rapportant toute la gloire à Dieu, à messire, comme elle disait dans son simple langage. Cette statue se ressent de l'époque où elle a été faite; elle date des dernières années de la Révolution, et ressemble un peu à une liberté de ce temps-làa ${ }^{20}$.»

Finalement, on ne peut que souscrire à la plupart de ces remarques lucides, pour méchantes qu'elles fussent. Très vite on souhaita à Orléans remplacer la statue par un monument plus noble. Il semble que dès 1820 il en fut question, en 1830 tout au moins Vergnaud-Romagnesi l'affirmait ${ }^{21}$. Il fallut attendre 1854 pour que l'arrivée de la Jeanne de Foyatier la repousse au sud de la Loire, à l'entrée du pont royal puis à son emplacement actuel. Mal-aimée mais connue! Raffet et Charles Ransonnette en répandirent vers 1840 une superbe version en buste dont les plumes s'envolent comme d'un chapeau de mousquetaire tout en insistant sur une féminité soulignée par une cuirasse sans équivoque ${ }^{22}$.

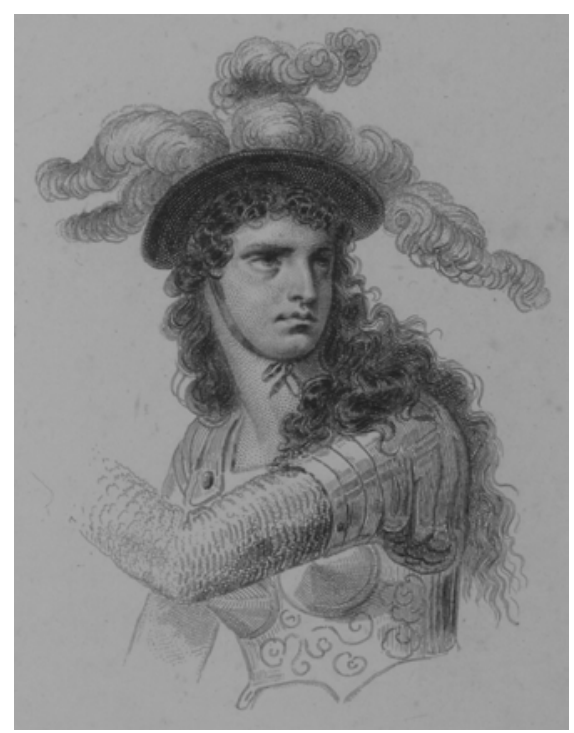

Buste de Jeanne d'Arc

Dessin de Raffet gravé par Ch. Ransonnette (C.J.A., 00.173.016)

${ }^{20}$ Feuilletons, Orléans, Alex Jacob, 1840, p. 195-196. Le voyageur était Joseph Etienne dit Jouy, membre de l'Académie française, qui passa à Orléans en 1827 (J. Lemaire, Notes pour servir à l'histoire de la statue de Jeanne d'Arc de Gois, Orléans, Houzé, 1945, p. 11).

${ }^{21}$ Vergnaud-Romagnesi, Histoire de la ville d'Orléans, Orléans, 1830, p. 372-378.

${ }^{22}$ C.J.A., inv. 00.173.163, 8 X $12.5 \mathrm{~cm}$, dans un cadre à double filet (Images de Jeanne d'Arc, Catalogue... op. cit., p. $83 \mathrm{n}^{\circ} 111$, avec une faute au nom du graveur). 
La statue avait une autre caractéristique, c'est son déséquilibre quand on la contemple de profil. Le commentateur cité plus haut l'avait relevé : «Elle a de l'expression, du mouvement, trop même, et un mouvement trop violent». Les basreliefs qui entourent le sommet du piédestal rappellent aussi cette fougue, de manière plus lisible sur les gravures du salon de 1803 que sur l'original. Jeanne attrape la bannière ennemie frappée du léopard, l'épée levée ${ }^{23}$. L'instant suivant est celui de la statue. L'élan fougueux fut réutilisé dans une caricature de 1815, comme modèle des amazones ${ }^{24}$.

La statuomanie historique commençait alors en Occident: on voulait faire soit à Versailles, soit au Louvre, le lieu de la célébration de l'histoire de France. Des concours eurent lieu: Évariste Fragonard, le fils du peintre, proposa en 1820 un modèle à réaliser ensuite en biscuit à Sèvres. Comment ne pas y retrouver à la fois le portrait des échevins en pied et l'œuvre de Gois fondus pour le thème appelé à faire fortune avec la Restauration: le sacre de Charles VII ? On a du mal aujourd'hui à imaginer l'invention de ce déguisement anachronique. Il en est de même avec le frontispice des Messéniennes de Casimir Delavigne, écrites en 1816 et illustrées une dizaine d'années plus tard par un des Devéria (Jacques, né en 1800 ou Eugène, né en 1805 ? $)^{25}$. La légende essaie de concilier la Pucelle en robe et béret avec les vers vigoureux du poète :

L'ange exterminateur bénit ton étendard;

Il met dans tes accents un son mâle et terrible,

La force dans ton bras, la mort dans ton regard

Et dit à la brebis paisible :

Va déchirer le léopard.

Ce qui se fait au prix d'une véritable censure: Jeanne a tout de l'agnelle et rien de la camarde, et l'ange exterminateur semble lui promettre, à elle, la palme du martyre plus que l'extermination à l'ennemi.

${ }^{23}$ C.J.A., inv. 83.4.90. Le motif est inspiré de la tapisserie du XVII ${ }^{\mathrm{e}}$ s. représentant la prise des Tourelles, sur un carton de Vignon. Une gravure de Lafitte sur le même modèle fut reprise pour un frontispice d'un livre de la même époque, gravé par Mlle Vallet (Images de Jeanne d'Arc, Catalogue... op. cit., p. 82, $\mathrm{n}^{\circ}$ 107) et pour une assiette de faïence de Choisy (Jeanne d'Arc, à la tête d'un corps de troupes, prend d'assaut le fort des Tournelles. Par son courage héröque, elle délivre Orléans de la fureur des Anglais, Orléans, coll. part.).

${ }^{24} \mathrm{Mgr}$ Le Nordez, Jeanne d'Arc racontée par l'image, les sculpteurs, les graveurs et les peintres, Paris, 1898, p. 395 : le titre de l'estampe est «Le serment des amazones françaises au pied de la statue de Jeanne d'Arc ». La Pucelle porte là un bouclier au coude du bras gauche et est en tabart court. Une Jeanne aussi pleine d'élan et en moins instable survint beaucoup plus tard avec la statue de Fournier (1896) à Beaugency et Patay, mais l'étendard fleurdelisé était alors celui de la Pucelle.

${ }^{25}$ C.J.A., inv. 00.173.087, frontispice de Devéria, gravé par Adèle Bacquoy dans C. Delavigne, Les Messéniennes, s. 1., s. d. La citation sous la gravure est extraite de la septième strophe de la quatrième Messénienne du livre I : «La vie de Jeanne d'Arc ». 


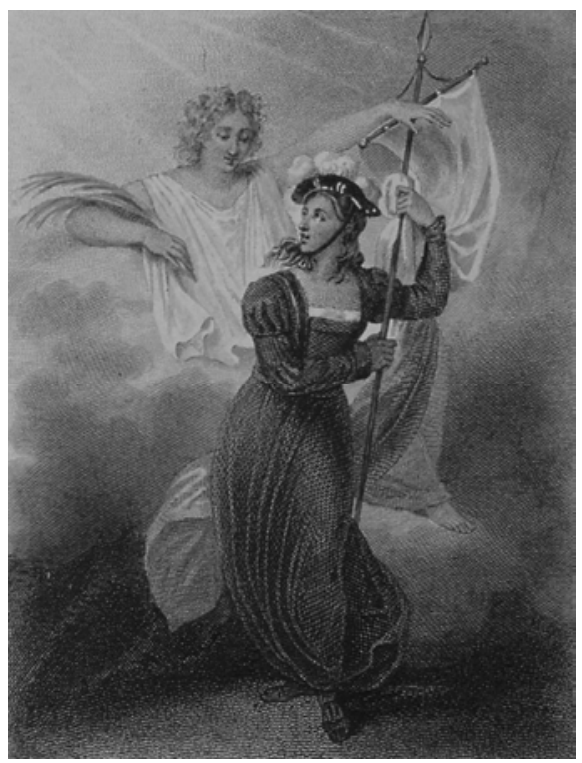

Frontispice pour les Messéniennes : Jeanne et l'ange exterminateur Dessin de Devéria gravé par Adèle Baquoy (C.J.A., inv. 00.173.087)

Il se révèle extrêmement difficile de faire coïncider plumes et velours avec la fureur de la guerre. Même dans l'évocation du théâtre du Vaudeville où l'on voyait opposer en 1812 à une Mademoiselle Rivière, fort décidée en paroles, à mi-chemin entre La Grande Mademoiselle et Hippolyte, reine des Amazones, des guerriers presque plausibles, dont le Pierre qui la poursuivait de ses assiduités ${ }^{26}$ :

Ne peut-on, parce qu'on est femme

Sauver sa patrie et son roi

Je sens à l'ardeur qui m'enflamme

Que Dieu m'appelle à cet emploi.

Oui tu me dois une victoire,

Dieu tu fais naître chaque jour

Assez de femmes pour l'Amour,

Qu'il en naisse une pour la gloire.

${ }^{26}$ C.J.A., inv. 79.4.28, 10.5 X 16 cm., Images de Jeanne d'Arc, Catalogue... op. cit., p. 119, $\mathrm{n}^{\circ}$ 183. L'étendard porte l'inscription «Espère en Dieu». En plus de l'épée et d'un petit bouclier, Jeanne est ceinte de l'écharpe blanche des maréchaux de France... 
Vers que semble reprendre la jolie Jeanne d'Arc à l'assaut aux yeux de velours mais aux gantelets de fer du soldat Gravier, inspiré par Vivien ${ }^{27}$.

Ainsi, l'Empire et la Restauration perpétuent-ils une tradition dans laquelle les modernes chevelus du romantisme vont s'engouffrer avant de reprendre leurs esprits et d'inventer la Jeanne historique sans panaches et sans robe.

En 1818, H. Lemaire propose une Vie de Jeanne d'Arc. Les illustrations offrent deux idylliques paysages (Rousseau, Goethe et Chateaubriand sont bien assimilés), et deux scènes de la vie de la Pucelle ${ }^{28}$. Sur celle de Jeanne d'Arc plantant son drapeau au siège d'Orléans, elle est en armure, mais un béret à plumes couronne encore un équipement presque historiciste. En revanche, l'entrevue avec un Baudricourt affublé d'une fraise et d'un pourpoint relève d'un costume de théâtre aussi absurde que celui de Mlle Rivière, quoique les plumes ne puissent figurer sur le bonnet de la petite paysanne.

En prison et enchaînée, Jeanne garde sa superbe coiffure devenue son signe caractéristique. Révoil en 1819 proposa au Salon la réponse méprisante de Jeanne aux menaces des compagnons de Warwick ${ }^{29}$ : elle porte bien six plumes au bonnet, mais la jupe est raccourcie en casaque et les jambes ont des chausses. On connaissait mieux en France depuis les travaux de L'Averdy, à la fin du XVIII ${ }^{\mathrm{e}}$ siècle, les textes du procès et l'insistance du tribunal à faire enlever ces indécents vêtements d'homme à la jeune fille. La leçon n'est cependant pas apprise par tous. On passe de Chasselat (1820), qui remet sa robe aux manches bouffantes à Jeanne, mais pose sur la table des juges un casque à plumet et fait glisser le béret sur la tête de Warwick ${ }^{30}$, à Ducis (1775-1847), qui dénude fort l'épaule de Jeanne enchaînée en prison et la fait haranguer, l'épée à la main, un casque empanachée ${ }^{31}$.

\footnotetext{
${ }^{27}$ Signée L. Gravier d'après Vivien, lithographie de A. Chalappe, rue de la Cerche à Orléans. Le sujet de Vivien (1657-1735) ne nous est pas connu.

${ }^{28}$ H. Lemaire, Vie de Jeanne surnommée La pucelle d'Orléans [...], ornée de quatre gravure en taille douce, Paris, Le Prieur, 1818. On remarque cependant que la gravure de l'entrevue a comme décor des panneaux à ogives, une porte à plis de serviette. Le mobilier troubadour était plus réaliste que le costume, décidément encore empreint du modèle des " échevins ».

${ }^{29}$ Pierre-Henri Revoil (1776-1842), Jeanne prisonnière à Rouen (1819), Rouen Musée des Beaux Arts, Jeanne d'Arc, Les tableaux de l'histoire,1820-1920, Catalogue de l'exposition, Rouen, mai-septembre 2003, cat. 5 et cat. 6.

${ }^{30} \mathrm{Mrg}$ Le Nordez, op. cit., p. 324. Sur cette lithographie de Chasselat (1782-1843), Warwick ressemble à François I ${ }^{\text {er }}$, les soldats rentrent des guerres d'Italie...

${ }^{31} \mathrm{Ibid}$, p. 328. Les gantelets et l'épée avec des pièces diverses de fer gisent au premier plan. Il est difficile de faire la part du rêve et de la représentation réaliste: les scribes du tribunal semblent prendre en note ce qui pourrait être un soliloque de prisonnière. Le tableau n'est connu que par une lithographie.
} 


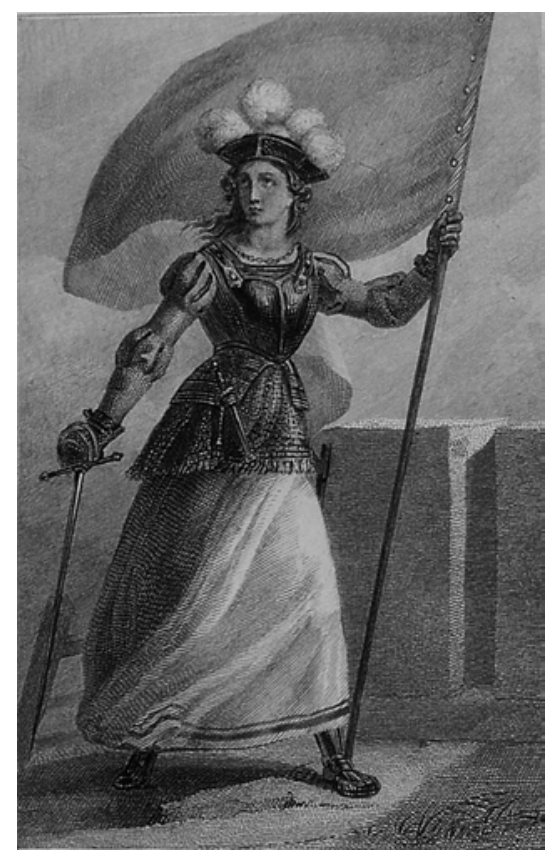

Jeanne à l'étendard

Dessin de Chasselat gravé par Bardet, 1820. (C.J.A. 00.173.12)

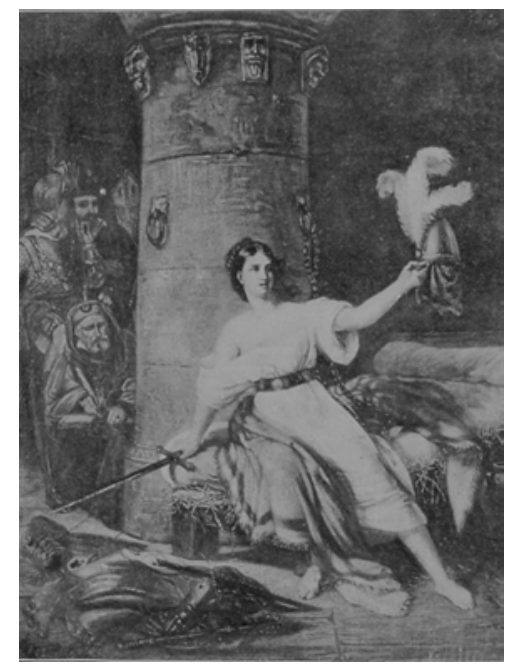

Captivité de Jeanne d'Arc

D'après un tableau de Ducis 
On peut constater que les cinéastes d'aujourd'hui sont presque moins imaginatifs que les peintres des salons romantiques. Il n'est pas jusqu'à la scène du bûcher que le fort sérieux - pour l'époque - manuel d'Anquetil $(\dagger 1805)$ qui ne propose dans l'édition de 1820 une Pucelle gentiment assise sur les bûches embrasées et le béret en tête.

Les années 1830 ne firent guère faire de grands progrès à la figure de Jeanne même si les historiens, en Allemagne tout particulièrement, reprenaient peu à peu les sources historiques des histoires nationales (Görres, Guizot, A. Thierry). A cette époque, Eugène Devéria ne trouvait encore rien d'autre à offrir pour une série des Têtes de femmes célèbres qu'un mélange d'armure, de longues boucles et de plumes où chacun pouvait reconnaître, sans même lire le titre, les attributs de l'icône de Jeanne d'Arc, Pucelle d'Orléans ${ }^{32}$. Le Congrès musical d'Orléans fit frapper en 1837 une médaille pour laquelle Charles Pensée (1799-1871) donna une Jeanne de profil qui reprenait le vêtement rituel, la chaîne de cou et les plumets ${ }^{33}$.

La révolution iconographique était cependant en marche, mais nul ne le savait, elle fut silencieuse. Paul Delaroche avait ouvert la voie de la simplicité et d'un romantisme fondé sur un certain réalisme en proposant en 1824 Jeanne d'Arc malade est interrogée dans sa prison par le cardinal de Winchester, grande toile où s'opposaient l'éclat de la grande robe écarlate d'un prélat à l'index impérieux et le petit visage encadré de bandeaux noirs de la prisonnière ${ }^{34}$. Le tableau, pourtant de très grande qualité, n'eut pas, semble-t-il, d'influence immédiate. On pouvait, en 1840, montrer encore en grand hors-texte L'entrée de Jeanne à Orléans où elle se présentait avec une armure, un caparaçon aux fleurs de lys et la fameuse coiffure à plume $^{35}$. En 1846, le frontispice anonyme du livre de Mlle A. Leclerc, Dieu, la France et Jeanne d'Arc ${ }^{36}$, ne concevait pas sans le célèbre béret se détachant sur un large étendard la remise par J. Talbot de ses armes à la victorieuse chevetaine (pour reprendre le terme de Christine de Pizan) des troupes de Patay.

On mesure avec cette dernière image, mièvre et étriquée, le choc que fut la modernité de la statue en marbre de Marie d'Orléans, placée dans la galerie historique du Palais de Versailles en 1837. Un dessin d'Hébert, sans doute le père, sculpteur (1804-1869) 37 , montre à la perfection l'intériorité de la prière, le poids du matériau de marbre, la simplicité des lignes saisies sous l'angle le plus flatteur, il faut le dire, et le raffinement qui consista à reconstituer une armure simple dont la jupe ne parût pas une concession à la pruderie du temps. Même le casque qui reposait sur les gantelets croisés avait perdu ses ornements ondoyants. Les cheveux plats

${ }^{32}$ C.J.A., inv. 00.173.083, 14 x 26 cm. Jeanne d'Arc, Pucelle d'Orléans, dessin de Devéria, lithographie de Lemercier.

${ }^{33}$ Médaille, bronze, diam. 4,5 cm, dessinée par Pensée, gravée par Caqué (Orléans, coll. part.).

${ }^{34}$ Musée de Rouen, inv. 982.6.1. Dossier du tableau, Rouen, 1994 ; Jeanne d'Arc, les tableaux de l'histoire, op. cit., cat. 9.

${ }^{35}$ A. E. de Saintes, Les anges de la terre, Paris, 1840, p. 169, hors-texte signé Sevilly (17 x 23 $\mathrm{cm})$.

${ }^{36}$ A. Leclerc, Dieu, Jeanne et la France, Orléans, Gatineau, 1846.

${ }^{37}$ C.J.A., inv. 77.54.6, dessin d'Hébert gravé par Aristide Louis. 
et courts, ou plutôt mi-longs de la tête penchée ne parurent pas scandaleux ${ }^{38}$, on pouvait tout accepter de la princesse si tôt disparue. Marie d'Orléans, avec les conseils et l'aide de ses professeurs, dont Ary Scheffer, et plus par son talent que par l'immédiat appui des orléanistes, a pratiquement tué une tradition alors vieille de deux siècles et demi. Parfaitement justifiée par les textes des procès et des chroniques, désormais accessibles grâce à la Société d'Histoire de la France et Jules Quicherat, l'audace de Marie d'Orléans trouva son public. La princesse ouvrit la porte à des représentations nouvelles et relégua béret, plumes et manches à crevés au rang des vieilles lunes. Sans bataille d'Hernani, la sculpture historique de Jeanne d'Arc avait fait sa révolution romantique, entraînant dessins, peintures et gravures à sa suite.

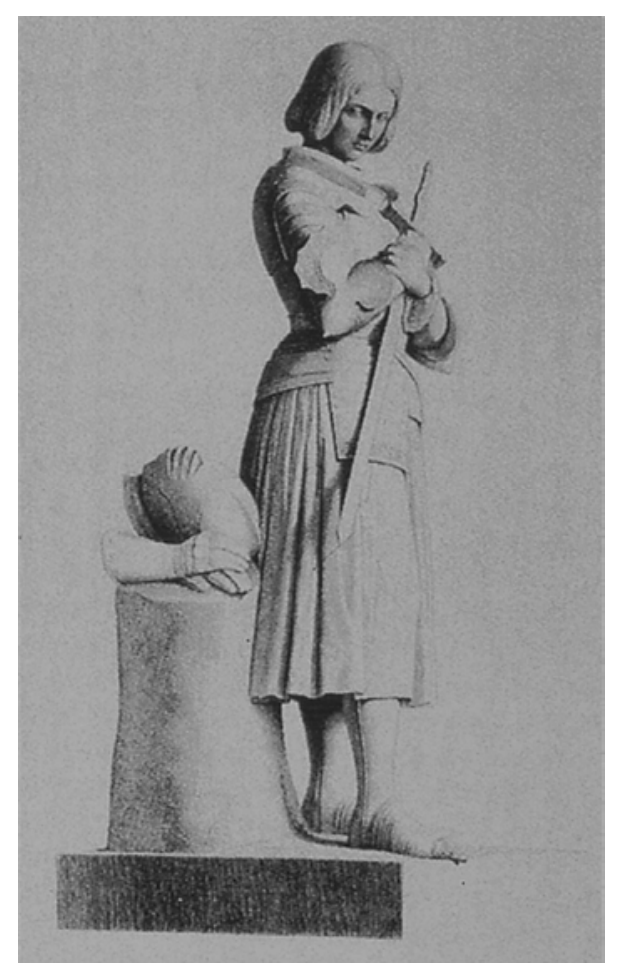

${ }^{38} \mathrm{Il}$ faut dire que la mode des bandeaux plats jusqu'au niveau des oreilles a grandement aidé à faire passer l'abandon des longues boucles libres. Henry Scheffer (1798-1862) avait en 1835 coupé mi-long les cheveux dans sa Jeanne d'Arc arrivant sur la place de Rouen: le prêtre qui l'avait trahie se jette à ses pieds et invoque son pardon du Musée d'Orléans (inv. MH15233). Ary Scheffer (1795-1858) reprit cette même coiffure pour son Entrée de Jeanne d'Arc à Orléans, 8 mai 1429 (1837), Musée national des châteaux de Versailles et des Trianons, représentation popularisée par la gravure. 